\title{
Developing a culture of Stewardship: how to prevent the Tragedy of the Commons in Universal Health Systems
}

Tim Wilson (1) , Gwyn Bevan (2), Muir Gray (3), Clara Day (4) and Joe McManners (5)

1 Nuffield Department of Primary Health Sciences, Oxford OX2 6GG, UK

2 London School Economics and Political Science, London WC2A 2AE, UK

3 Nuffield Department of Surgery, Oxford OX3 9DU, UK

4 University Hospitals Birmingham, Birmingham B15 2GW, UK

5 Manor House Surgery, Headington, Oxford OX3 9BL, UK

Corresponding author: Tim Wilson. Email: tim.wilson@phc.ox.ac.uk

Published in the Journal of the Royal Society of Medicine; 2020, Vol. 113(7): 255-261

DOI: 10.1177/0141076820913421 


\section{Developing a culture of Stewardship: how to prevent the Tragedy of the Commons in Universal Health Systems}

\section{Introduction}

Making best use of finite resources for populations is a driving force behind the trend for "accountable care" globally (e.g. Integrated Care Systems in the English NHS), but this is increasingly challenging with pressure on budgets and workforce. ${ }^{i}$ ii Sadly, collective management of resources to optimise outcomes for the whole population is far from the case in most countries and the 'Tragedy of the Commons'(see box 1), helps us understand

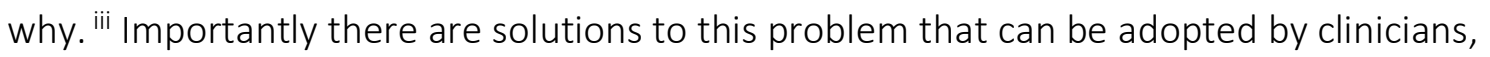
patients and the public and health leaders.

The English economist William Forster Lloyd published a pamphlet in 1833 that described the behaviour of shepherds sharing a common parcel of land on which they grazed their sheep. He suggested that if a shepherd put more than his allotted number on the common, overgrazing would result. The shepherd might benefit, but the result was loss of the grazing to others. But since it is a rational decision to add more animals, most shepherds would do the same, so the common could be depleted or even destroyed, to the detriment of all. This pamphlet was then developed by Garrett Hardin into the economic theory, the Tragedy of the Commons, published in Science in 1968

Box 1 Tragedy of the Commons

What are the 'Commons'?

Like common grazing land, universal health systems include shared finite resources that should be used for the common good of the population covered. Resources include money, workforce (both the people and their time), organisational priorities, carbon (and physical materials) and physical infrastructure. Understanding the Tragedy of the Commons and how to avoid it is important - it impacts clinicians and the people they serve.

Is the Tragedy of the Commons seen in health systems?

Universal health systems are defined by some key principles. The NHS Constitution, for instance, emphasises how care should be based on need, available to all, agnostic of organisational boundaries and represent value for money. But in England, at least, this does not seem to be the case: variations in spend and activity are far greater than can be explained by need; iv inequity of access is common, including for elective surgery, diabetes 
care and access to specialists; ${ }^{v}$ vi vii viii ix and variation in the value for money is notable with a ten-fold difference observed in resources required to improve outcomes. ${ }^{x}$

Why do universal health systems experience the 'Tragedy of the Commons'?

Funding is provided to jurisdictions for a given population as a capitated budget from insurance premiums or government revenues (the common financial resource). However, in most countries the common resources are then split amongst multiple providers (e.g. primary care, social care, hospital care, mental health services) and sometimes through different purchasers (e.g. for social care, aspects of health). ${ }^{\mathrm{i}}$ Thus, the common resource is fragmented into multiple plots, losing visibility of the whole. Indeed, the fragmented plots are further subdivided into departments serving different groups (e.g. surgery, or gastrointestinal surgery, or lower gastrointestinal surgery) or providing different functions (e.g. laboratories). The commons are no longer common, but "bits" (see figure 1). The result is competition for resources, with no sense of responsibility for the whole common resource.

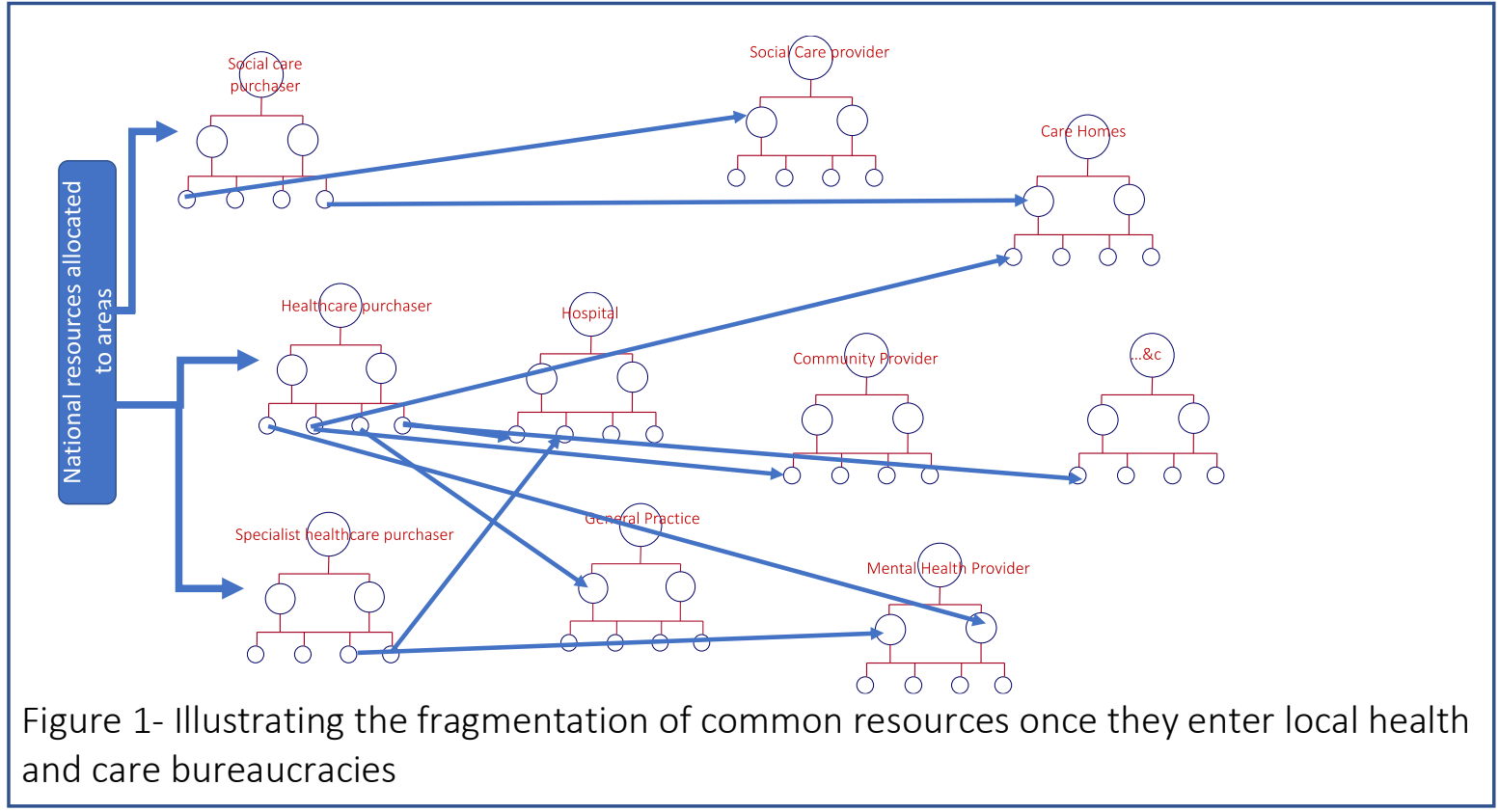

The Office for Budget Responsibility outlined in their report on health spending in the UK that the principal demnd on the Commons is not demographic change (e.g. ageing) but trends driven by clinical practice (see figure 2 ), the:

- Uncritical adoption of innovation;

- Increases in the volume of clinical practice. 


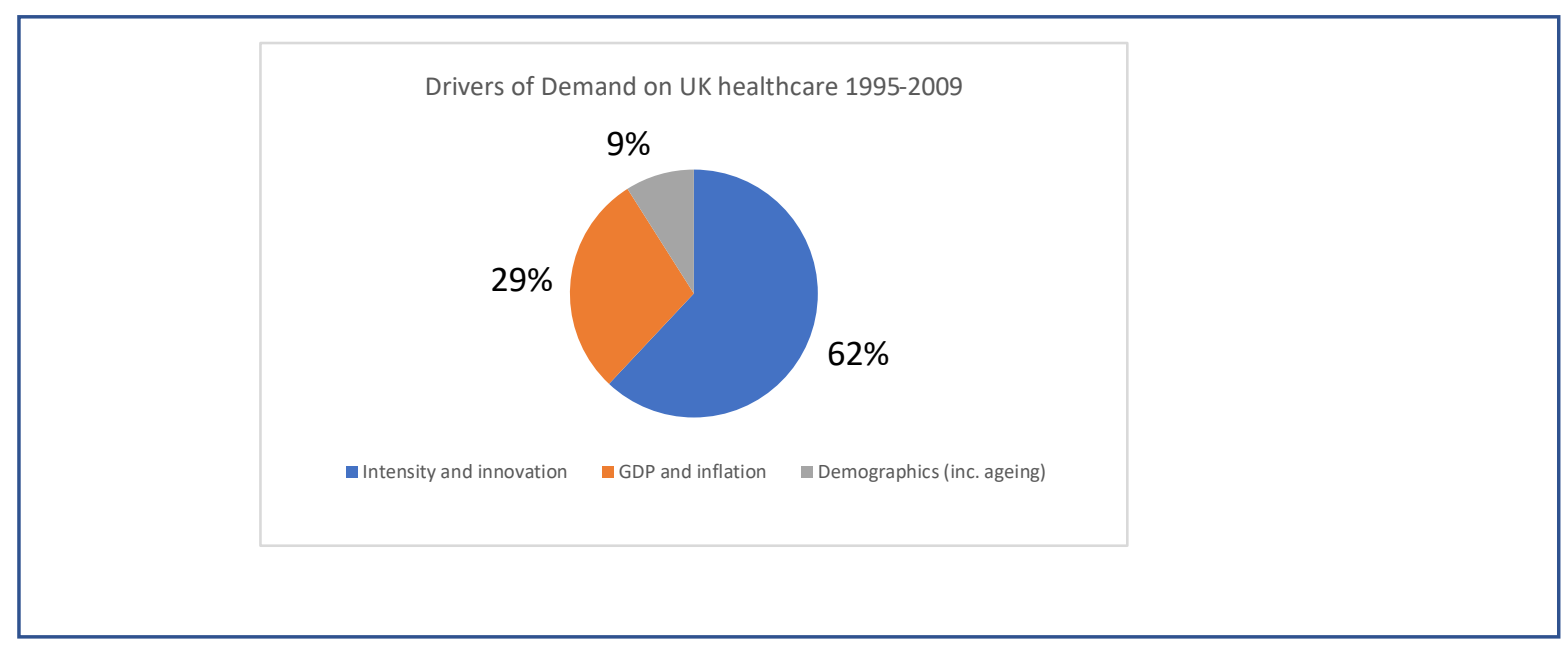

Figure 2: Drivers of demand in UK health system from Office of Budget Responsibility ${ }^{x i i}$

Memorably, David Eddy, in describing how clinicians simply do more and more, coined the phrase:

"The relentless increase in the volume and intensity of clinical practice". ${ }^{\text {iii }}$

This effect is broadly the same across all OECD countries. Almost never do these increases replace a lower value intervention or waste, but instead added to the demands on the common resources. The fragmentation of the common resource means that these increases happen in a largely uncoordinated manner.

McGinnis, Berwick and Ham have all seen the flaw in this from a population perspective and called for the creation of "regional health commons", the aggregation of all resources being deployed for a given population group in a region into a single common pool. ${ }^{\text {iv } x v \text { xvi }}$

\section{Avoiding the Tragedy of the Commons}

Nobel prize winning economist, Elinor Ostrom outlined in her work a set of ten principles to solve the Tragedy of the Commons, distilled from analysis of numerous case studies around the world (see box 2). 
Leading the commons

1. Commons need leadership with moral authority

Defining the commons

2. Commons need clear boundaries

3. Commons need clear aims

Organising the commons

4. Commons need the right to organise themselves

5. Everyone who makes use of the resources should have a role in decision making.

6. Commons often work within nested networks

Managing the commons

7. Rules for resource use should match local context

8. The use of resources and adherence to rules should be monitored

9. Failure to meet the rules leads to graduated sanctions

10. Unresolved conflicts need an easy means of resolution

Box 2 Ten principles for managing common resources, adapted from Ostrom for healthcare by McGinnis, grouped by the authors. ${ }^{x v i i}$

\section{How might the principles apply to healthcare to provide solutions to this problem? \\ In creating "regional health commons", McGinnis, Berwick and Ham recognised that Ostrom's principles might be applied to healthcare. Here we suggest how those principles may be applied to universal health care.}

\section{Leading the commons}

In 2014 the Academy of Medical Royal Colleges in the UK outlined a clinical culture whereby: "As responsible stewards, doctors can provide a more effective use of constrained economic and environmental resources." "xviii Further, in the UK, the General Medical Council advice on being a licensed medical practitioner is that Doctors when making decisions about 'using resources' must take account of "your responsibilities towards your patients and the wider populations". ${ }^{\text {ix }}$

These responsibilities recognise that every single clinician, as a direct provider of care, is making hundreds of resource decisions daily. This is one reason they should be central to managing the commons. But also a good reason for clinicians to be central to the development of a culture of stewardship, so resources are used to improve population and individual outcomes. The importance of leaders able to bring together people from disparate professional backgrounds and rally them to a common purpose cannot be under-estimated. In this regard, it is the leadership role of creating the right culture, stewardship, that matters most. ${ }^{x x}$ 
Since universal health care is for the benefit of the whole population, boundaries of the commons should be defined by population groups, not institutions. The boundaries need to be defined in two ways: first, by geographical area; and second, segmented by a common need.

Segmentation by need is critical, otherwise the commons are too heterogenous to manage. Segmentation should recognise the importance of people with complex needs (e.g. people who are frail), people with needs defined by a key stage in life (e.g. death), people who are healthy whose need is to remain so, and people with needs caused by significant conditions

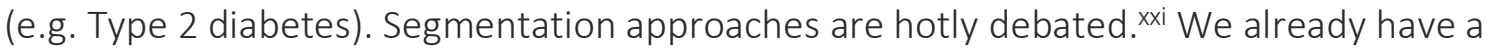
globally agreed segmentation approach by disease, the International Classification of Diseases (ICD). However, ICD fails to recognise the complexities of co-morbidities and other population groups with specific needs. Therefore, many health systems are using approaches like "Bridges to Health". xxii Bridges to Health is planned to be rolled out across the NHS in England during 2020.

Common resources must be quantified. For each population segment, all the resources should be identified (based on historical use). This way the fragmented "bits" of financial resources, described above, are once again aggregated into a common resource. Over time, those common resources should be re-allocated from one population segment to another based on need but in the short term it is more important to develop systems thinking and a culture of stewardship.

Each population group needs clear aims. Some aims should reflect the principles of the health system as a whole (e.g. the NHS Constitution for England would suggest that equity and value for money are clear aims for managing the commons in England). Other aims should reflect the outcomes that matter to the people with a need (e.g. remaining independent and confident for people who are frail).

\section{Organising the commons}

Organising the commons by geographical population segments does not require structural change. Indeed, health systems require institutions like hospitals and primary care clinics. Collaborative networks should be established, to manage the commons for population segments, and to work across institutional boundaries. All key people who use, or strongly influence the use of, resources must be included in decision making, including patients and the public, specialist and generalist clinicians.

Networks working across institutions, especially with a set of mutually agreed principles under which the network operates, are a proven way to organize the commons by segments. ${ }^{\text {xiii } x x i v}$ Especially for complex systems like health and care. ${ }^{x \times v}$

Nesting of networks with adjacent networks or as part of a bigger grouping of networks, as suggested by Ostrom, will allow three things to happen:

- Comparisons, sharing and learning between networks;

- Sharing of scarce resources (e.g. skilled workforce, equipment); and

- Pooling of financial risks (where appropriate). 
At the heart of Ostrom's research was the realisation that local communities determined their own set of rules and means of enforcement. Local networks would therefore need to have the authority (given by their constituent institutions) to invest, disinvest and reinvest allocated resources with the aim of improving outcomes for their population segment in their community. The network determines the operational response informed by local context.

Governance and 'rules' for achieving the aims are important, the network needs to monitor how resources are being used (e.g. is an agreed local pathway being followed?). Alongside celebrating effort and successes, a locally appropriate means of managing network members who are not following the rules needs to be established and collective ways of managing conflicts. This can include proven approaches such as; sharing and publishing performance; training; peer to peer feedback and others. ${ }^{x x v i}$

If collective decisions are made to disinvest and reinvest resources, including "fixed" assets such as beds, major equipment or staff, there needs to be a managed process of resource reallocation.

An important principle in managing the commons is for those doing so to see the benefit of improving value in resource use, the ability to reinvest within their population. An appeal to clinicians to find 'savings' to reduce an overspend is likely to be ineffective, whereas a challenge to increase high value activity, by shifting resources from lower value activity will stimulate thought and engagement

Being held accountable

In addition to the ten principles, in the public sector there is the need to be accountable. Like all organisations making decisions about the use of public resources, networks need to be accountable to the population they serve as well as to the bureaucractic structure. A starting point might be a system of public reporting on the outcomes they achieve and associated resource use. Evidence suggest is likely to promote further improvements. ${ }^{x x v i i}$ Accountability also means that autonomy is not inevitable: sustained poor performance compared to other networks, alongside an inability to learn, would likely trigger stronger interventions (e.g. direct control).xxviii

What does managing the commons look like?

Fictional Barsetshire health and care system (figure 3), covers a population of 874,231 people and is co-terminus with the Council which provides social care. There are three distinct 'places' with sizeable towns; Barchester, Hogglestock and Silverbridge, each of which has a District Council and two of which have a hospital.

In Silverbridge, providers are working together to care for the population of 324,755 . They have established networks across their organisations to cover all the major population segments, including people who are frail (number $=5634$ ), end of life, adults with established type 2 diabetes and so forth. Networks include relevant clinicians from all the providers, patient representatives and the public, and managers. 


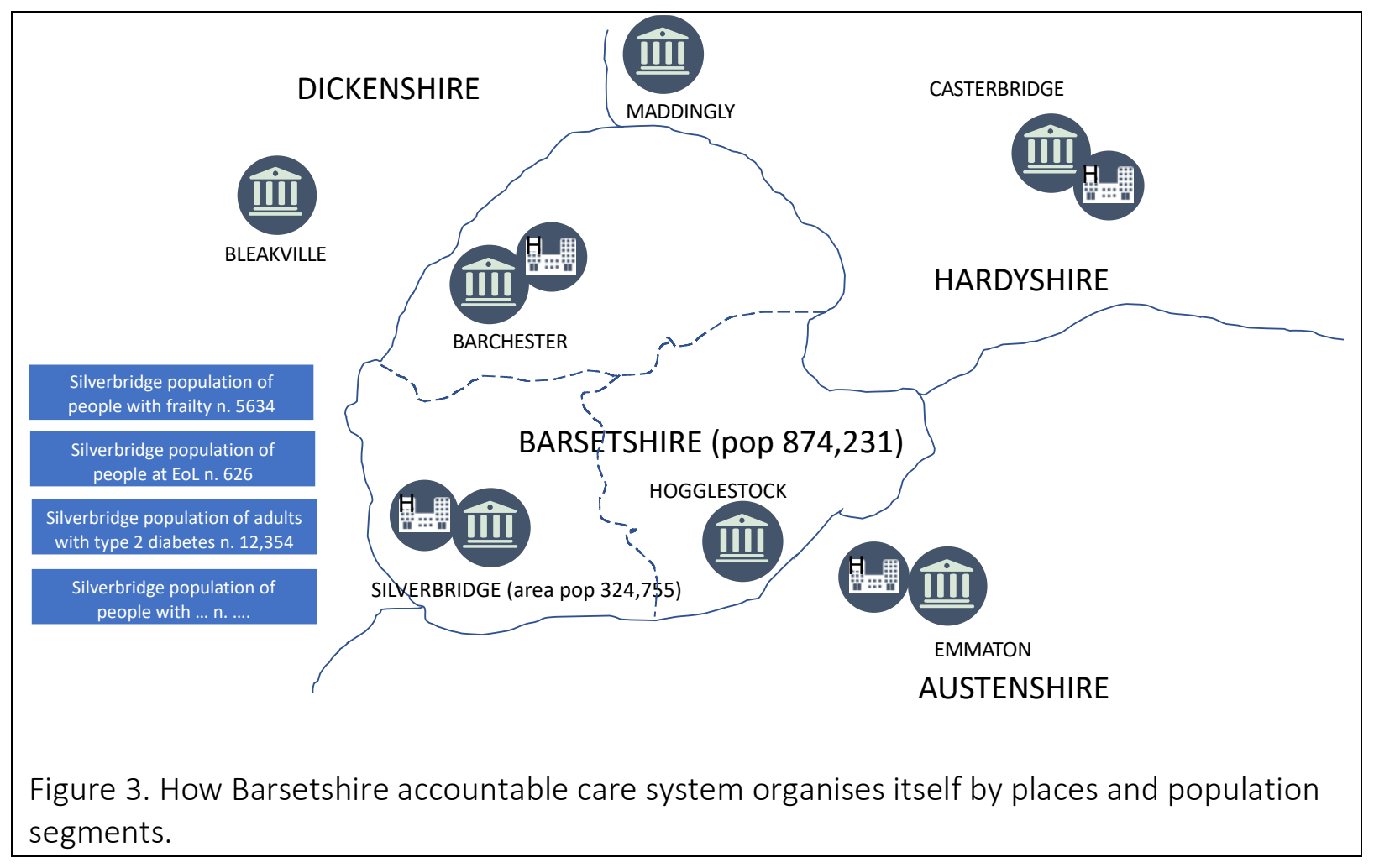

Each network knows the outcomes they need to achieve as these have been developed through public consultation by Barsetshire Health and Care System. They also know the resources they have available as these have been allocated by the Barsetshire Health and Care System, using an approach that moves from historical spend to a needs-based allocation (figure 4).

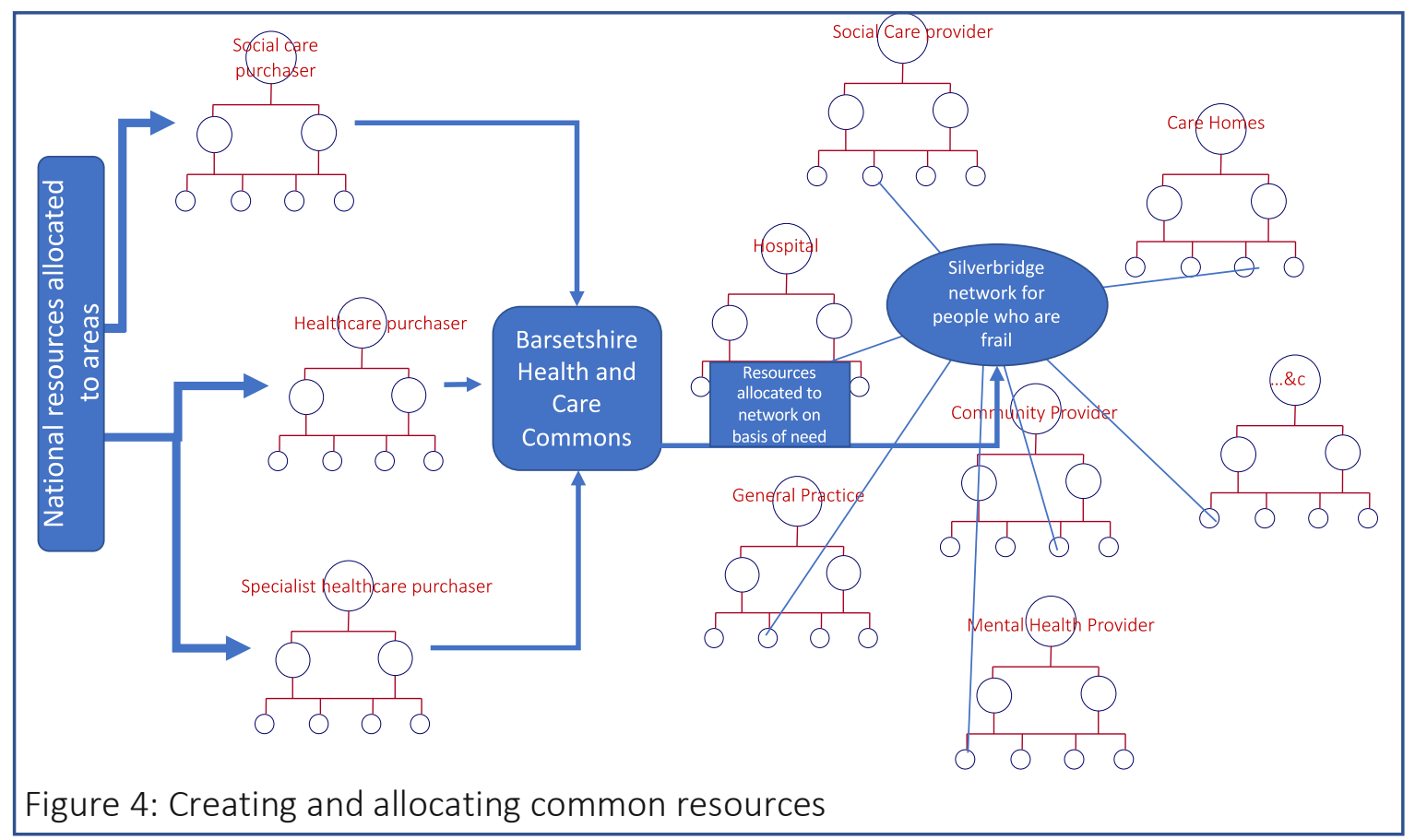


Silverbridge provider alliance supports the investment, disinvestment and reinvestment of resources across all their networks. They oversee the development of the networks, provide financial, analytical and other managerial support and performance manage as required (noting the importance of autonomy). They also manage the sharing of high cost assets across networks in Silverbridge. But each network is ultimately responsible for managing the common pooled resource for the population they serve in a sustainable fashion.

There are identical networks in Barchester and Hogglestock. Barsetshire Health System encourages each of these networks to learn from each other and publishes the performance of each network in the Barsetshire Gazette each year. They also intervene in persistently failing networks.

\section{Conclusion}

We have argued that the Tragedy of the Commons operates in universal health systems impinging on population health outcomes. Some countries, like England, are seeking to remove structural barriers to managing the commons. But, the evidence is clear. Structural change has limited positive impact and is hugely distracting. xix Countries with the structures England aspires to, still suffer the Tragedy of the Commons. Thus, to manage the commons, establishing a culture of stewardship must come first and foremost, with structural and other changes in support. By incorporating Ostrom's principles there is a clear mechanism for culture change at the clinical and individual level so often absent in reforms.

Continuing with today's approach is unsustainable: increased costs, greater pressures on staff, continuing inequity and the likely degradation of a common good. It is no understatement to say change will not be easy, including major ways to the way finances are managed across the system. If clinicians, alongside patients and the public, adopt a culture of stewardship, create networks and lead reinvestment from wasted resources to higher value interventions, then we are optimistic that we can preserve much that is good in universal health systems.

\section{Acknowledgements}

We thank Professor Chris Ham and Dr Carrie McEwen for comments and Professor Al Mulley and Hugh Alderwick for providing us with the original idea.

\footnotetext{
i McClellan, M., Kent, J., Beales, S. J., Cohen, S. I. A., Macdonnell, M., Thoumi, A., ... Darzi, A. (2014). Accountable Care Aro und The World: A Framework To Guide Reform Strategies. Health Affairs, 33(9), 1507-1515.

ii Kleinert, S., \& Horton, R. (2017). From universal health coverage to right care for health. Lancet (London, England), 390(10090), 101-102.

iii Hardin, G. (1968). The Tragedy of the Commons. Science, 162(3859), 1243-1248.

iv Wennberg International Collaborative Medical Practice Variation Publication List available at http://wennbergcollaborative.org/uploads/documents/Corallo Systematic review MPV in OECD Appendix HPol 131.pdf

v Sheringham, J., Asaria, M., Barratt, H., Raine, R., \& Cookson, R. (2017). Are some areas more equal than others? Socioeconomic inequality in potentially avoidable emergency hospital admissions within English local authority areas. Journal of Health Services Research \& Policy, 22(2), 83-90.

vi Judge, A., Welton, N. J., Sandhu, J., \& Ben-Shlomo, Y. (2010). Equity in access to total joint replacement of the hip and knee in England: cross sectional study. BMJ, 341, c4092.

vii Asthana, S., Moon, G., Gibson, A., Bailey, T., Hewson, P., \& Dibben, C. (2018). Inequity in cardiovascular care in the English National Health Service (NHS): a scoping review of the literature. Health \& Social Care in the Community, 26(3), 259-272.
} 
viii Khanolkar, A. R., Amin, R., Taylor-Robinson, D., Viner, R. M., Warner, J. T., \& Stephenson, T. (2016). Young people with Type 1 diabetes of non-white ethnicity and lower socio-economic status have poorer glycaemic control in England and Wales. Diabetic Medicine, 33(11), 15081515.

ix Willi, S. M., Miller, K. M., DiMeglio, L. A., Klingensmith, G. J., Simmons, J. H., Tamborlane, W. V., ... T1D Exchange Clinic Network. (2015). Racial-Ethnic Disparities in Management and Outcomes Among Children With Type 1 Diabetes. PEDIATRICS, 135(3), $424-434$.

x Martin, S., Rice, N., \& Smith, P. C. (2008). Does health care spending improve health outcomes? Evidence from English programme budgeting data. Journal of Health Economics, 27(4), 826-842.

${ }^{x i}$ Gray, M. (2017). Population health: the third dimension. Journal of the Royal Society of Medicine, 110(2), 54-56.

xii Licchetta, M., \& Stelmach, M. (2016). Fiscal sustainability analytical paper: Fiscal sustainability and public spending on health.

xiii Eddy, D. M. (1993). Three Battles to Watch in the 1990s. JAMA: The Journal of the American Medical Association, $270(4), 520$.

xiv McGinnis M, Brink C. 2012. Shared Stewardship of a Health Commons: Examples and Opportunities from Grand Junction, Colorado. A

White Paper Prepared for the Alliance of Community Health Plans (http://www.achp.org/)

xv Berwick, Donald M. 2009. "Squirrel," Plenary Address, 21st IHI Annual National Forum on Quality Improvement in Health Care, Orlando,

FL: December 8, 2009. http://www.scribd.com/doc/24191521/Plenary-Text-Final

xvi Ham C. 2019 Triumph of the Commons. Blog. Available at https://www.hsj.co.uk/integration/integrated-care-systems-could-deliver-thetriumph-of-the-commons/7026408.article

xvii McGinnis, M. D. (2013). Caring for the Health Commons: What it is and Who's Responsible for it. SSRN Electronic Journal.

https://doi.org/10.2139/ssrn.2221413

xviii Protecting Resources, Promoting Value. Academy of Medical Royal Colleges, 2014

xix https://www.gmc-uk.org/ethical-guidance/ethical-guidance-for-doctors/leadership-and-management-for-all-doctors/planning-using-andmanaging-resources

xx Groysberg B et al. The Leader's Guide to Corporate Culture (2018) Harvard Business Review, January- February,

${ }_{x \times i}$ Chong, J. L., Lim, K. K., \& Matchar, D. B. (2019). Population segmentation based on healthcare needs: a systematic review. Systematic Reviews, 8(1), 202.

xxii LYNN, J., STRAUBE, B. M., BELL, K. M., JENCKS, S. F., \& KAMBIC, R. T. (2007). Using Population Segmentation to Provide Better Health Care for All: The "Bridges to Health" Model. The Milbank Quarterly, 85(2), 185-208.

xxiii Child J. Organization. Contemporary Principles and Practice. Blackwell Publishing, 2005

xxiv Harvard Business Review Analytic Services. (2019). Testing Organizational Boundaries to Improve Strategy Execution Retrieved April 12, 2019, from https://hbr.org/sponsored/2019/04/testing-organizational-boundaries-to-improve-strategy-execution

xxv Plsek, P. E., \& Wilson, T. (2001). Complexity, leadership, and management in health organisations. BMJ (Clinical Research Ed.), 323(7315), $746-749$.

xxvi Grol, R., Baker, R., \& Moss, F. (2002). Quality improvement research: understanding the science of change in health care. Quality \& Safety in Health Care, 11(2), 110-111.

xxvii Bridgewater, B., Grayson, A. D., Brooks, N., Grotte, G., Fabri, B. M., Au, J., ... North West Quality Improvement Programme in Cardiac Interventions. (2007). Has the publication of cardiac surgery outcome data been associated with changes in practice in northwest England: an analysis of 25,730 patients undergoing CABG surgery under 30 surgeons over eight years. Heart (British Cardiac Society), 93(6), 744-748.

xxviii Bevan, G. (2015). Improving public services through ambitious targets and tough sanctions for failure. Retrieved from http://eprints.Ise.ac.uk/62854/

xxix Smith, J., Walshe, K., \& Hunter, D. J. (2001, December 1). The "redisorganisation" of the NHS. British Medical Journal. 